\title{
PREFERÊNCIA POR ALIMENTOS SEGUROS: ESTUDO DE CASO DO CONSUMO DE MORANGO EM UM MUNICÍPIO DE MINAS GERAIS ${ }^{1}$
}

\author{
Djalma Adão Barbosa Júnior ${ }^{2}$ \\ Airton Lopes Amorim ${ }^{3}$ \\ Franciane Rocha de Faria ${ }^{4}$ \\ Silvia Eloiza Priore \\ Marília Fernandes Maciel Gomes ${ }^{6}$
}

\begin{abstract}
Resumo: Apesar do esforço governamental em monitorar resíduos de agrotóxicos em frutas e hortaliças, alguns produtos, como o morango, continuam apresentando níveis acima do permitido por lei. Desta forma, este estudo se propôs avaliar a preferência dos consumidores, estimada por meio da disposição a pagar (DAP), por morangos produzidos sem a utilização de agrotóxicos, além de analisar o efeito da informação a respeito dos malefícios à saúde decorrentes da ingestão de agrotóxicos sobre a DAP. Foram aplicados dois questionários semiestruturados, um contendo informação acerca dos efeitos adversos à saúde ocasionados pela ingestão de agrotóxicos e outro sem essa informação. Estimou-se a DAP individual por meio da técnica de referendo simples, ou pergunta fechada. Utilizou-se o método de valoração contingente na obtenção dos dados primários da pesquisa e na análise estatística, o modelo Logit. Os resultados mostraram que os consumidores deste município estão dispostos a pagar, aproximadamente, duas vezes mais pelo morango produzido sem agrotóxico, isto é, $\mathrm{R} \$ 6,68$, quando não informados dos malefícios da ingestão de agrotóxicos. Já a DAP dos consumidores que tiverem acesso a essa informação aumentou aproximadamente $16 \%$, chegando a RS 7,76. Demonstra-se, assim, a preferência dos consumidores da região por produtos sem agrotóxicos.
\end{abstract}

Palavras-chave: segurança alimentar; morango; demanda de alimentos

1 Recebido em: 31/08/2012; Aceito em: 29/01/2013.

2 Mestre em Economia Aplicada pelo Departamento de Economia Rural da Universidade Federal de Viçosa - DER/UFV. Bolsista CAPES. E-mail: djalma_ea@yahoo.com.br.

3 Doutorando em Economia Aplicada pelo Departamento de Economia Rural da Universidade Federal de Viçosa - DER/UFV, Bolsista do CNPq. E-mail: aimorim2007@yahoo.com.br.

4 Doutoranda em Ciência da Nutrição pelo Departamento de Nutrição e Saúde da Universidade Federal de Viçosa - DNS/UFV. Bolsista CAPES/REUNI. E-mail: franciane.faria@ufv.br.

5 Docente do Departamento de Nutrição e Saúde da Universidade Federal de Viçosa -DNS/UFV. E-mail: sepriore@ufv.br.

6 Docente do Departamento de Economia Rural da Universidade Federal de Viçosa DER/UFV. E-mail: mfmgomes@ufv.br 


\begin{abstract}
Besides governmental effort in monitoring pesticide residues in fruits and vegetables, some products, like strawberry, continue presenting pesticide residue levels above the specified by law. Therefore, this study evaluated the consumer preference, estimated by the willingness to pay (WTP), for strawberry produced without pesticide, and evaluated the effect of information regarding the health injuries caused by pesticide ingestion over the WTP. It was applied two semi-structured questionnaires, on with information regarding the adverse health effect due to pesticides ingestion and other without that information. It was estimated the individual WTP by the simple referendum, or closed question. It was used the contingent evaluation method on the acquisition of primary data and for the statistical analysis the Logit model. The results show that consumers of this municipality have WTP, approximately, twice larger by the strawberry produced without pesticides, that is, $\mathrm{R} \$ 6,68$, when not informed about the health problems of pesticide ingestion. On the other hand, the WTP of consumers that had access to information raised approximately $16 \%$, reaching RS 7,76. It is demonstrated, therefore, the preference of this region consumers by products without pesticides.
\end{abstract}

Keywords: food safety; strawberry; food demand

\title{
1. Introdução
}

Na medida em que os países se desenvolvem - em termos de renda e educação - a população tende a modificar suas preferências, passando de uma necessidade básica de aquisição de energia para a escolha de alimentos mais nutritivos e saudáveis. Uma característica que passa, então, a ser mais valorizada é a segurança do alimento (HENSON E CASWELL; 1999).

Embora possa existir demanda por um alimento seguro, esta característica é dificilmente observada pelo consumidor, já que na maioria dos casos o indivíduo somente percebe que um alimento está contaminado após efetivar sua compra (SEGERSON; 1999), ou mesmo sua ingestão. Dessa forma, o mercado de alimentos é caracterizado por informação imperfeita, isto é, o consumidor não tem todas as informações necessárias para escolher o produto de acordo com suas preferências. Por outro lado, normalmente o produtor tem esta informação, ou seja, existe o que se chama de assimetria de informação. 
O caso de alimentos contaminados com agrotóxicos é um exemplo típico da ocorrência de falhas de mercado, que apresentam custos amplos: custos individuais, isto é, diretamente para a saúde e o bem-estar do indivíduo, já que o consumo de agrotóxico traz efeitos adversos sobre a saúde, como a ocorrência de vários tipos de câncer, neuropatias, nefropatias, doenças hepáticas, intoxicações agudas, abortos e malformações fetais; e custos sociais, relacionados aos gastos com tratamentos no sistema de saúde que, para a maioria da população, são custeados pelo Sistema Único de Saúde.

O consumidor que conhece as consequências do consumo de agrotóxicos sobre a saúde tende a estar disposto a pagar mais por alimentos saudáveis. Dessa maneira, a informação de que o consumidor dispõe também influencia sua decisão em comprar ou não certos produtos, como mostrado por Prescott e Young (2002) em estudo que verificou o efeito da informação relativa à existência de glutamato monossódico sobre a disposição a pagar (DAP) por sopas, e mostrado por Rozan, Stanger e Willinger (2004), em estudo que verificou a influência de informação sobre a existência de metais pesados e suas consequências para a saúde na DAP em relação ao pão, maçã e batata.

Uma ação do governo brasileiro para atenuar as falhas de mercado com relação à utilização de agrotóxicos é o Programa de Análise de Resíduos de Agrotóxicos em Alimentos (PARA). Este programa, vinculado à Agência Nacional de Vigilância Sanitária (ANVISA), entrou em atividade em 2003 e tem como objetivo avaliar a qualidade dos alimentos comercializados por meio da análise de resíduos de agrotóxicos. Dados do PARA mostraram que, em 2010, 28\% das amostras de frutas e hortaliças foram consideradas insatisfatórias por apresentarem resíduos de produtos não autorizados ou autorizados, mas acima do limite máximo recomendado, com 59,5\% dessas culturas apresentando pelo menos um ingrediente ativo em situação irregular, como o acefato, substância banida em diversos países. Entre os alimentos analisados, o morango é um dos que apresentam, de forma recorrente, elevados níveis de resíduos de agrotóxicos (ANVISA, 2011). 
O objetivo do estudo foi estimar a DAP dos consumidores de um município da Zona da Mata Mineira por morangos produzidos sem agrotóxicos e o impacto do esclarecimento dos efeitos adversos dos agrotóxicos sobre a DAP. O interesse no comportamento dos consumidores da Zona da Mata Mineira se justifica pela sua proximidade geográfica com a maior região produtora de morango do estado, que é o sul de Minas Gerais (MADAIL et al., 2013), bem como pelo fato de o município de Viçosa/MG apresentar a segunda maior renda per capita da Zona da Mata Mineira (FJP, 2010), o que pode favorecer a demanda potencial por alimentos diferenciados e saudáveis.

Segundo levantamento do Instituto Brasileiro de Geografia e Estatística (IBGE), os dados da produção de morango vêm mostrando crescimento nos três últimos anos. Em Minas, a produção do fruto está concentrada em 27 municípios, 20 localizados na região Sul, que respondem por 95\% da safra. As lavouras que respondem pelos 5\% restantes da produção mineira estão localizadas nas regiões Central e Alto Paranaíba. Os municípios que lideram o ranking mineiro são Bom Repouso, Pouso Alegre e Estiva, responsáveis por 57,3 mil toneladas, ou $72,2 \%$ da safra estadual.

\section{Metodologia}

A DAP pelo morango sem agrotóxico foi determinada pela análise de valoração contingente, uma metodologia baseada em pesquisa survey, que proporciona aos entrevistados tomar decisões econômicas relacionadas ao consumo de bens não disponíveis no mercado (CARSON, FLORES, MEADE; 2001).

Para obter a DAP, cria-se um mercado hipotético, no qual o entrevistado é um consumidor potencial que revelará sua preferência pelo bem em função de suas respostas perante as DAPs, ou disposições a adquirir propostas pelo entrevistador. Conforme salientado por Mitchell e Carson (1989), o mercado hipotético deve apresentar-se o mais próximo possível de um mercado real, de forma que o entrevistado tenha acesso 
a informações detalhadas acerca do nível de provisão do bem, possíveis substitutos e forma de pagamento.

O mercado hipotético apresentado aos entrevistados refere-se à venda de uma embalagem de 300 gramas de morango sem agrotóxico a um preço determinado. Apesar de existirem produtos no mercado com tal característica, o mercado de morango sem agrotóxico ainda é muito incipiente, sendo necessária a obtenção de informações sobre seu consumo.

Os valores propostos como preços para a embalagem de 300 gramas do morango sem agrotóxico foram obtidos em pesquisas de mercado sobre o preço médio das embalagens de morangos produzidos do modo convencional (com agrotóxico) e entrevistas com especialistas sobre esse assunto, a fim de que pudessem apontar a formação de um cenário confiável sobre o possível intervalo de preços. A pesquisa de mercado indicou a venda da embalagem de morangos comuns por até $\mathrm{R} \$ 2,00$, apontando preço médio de $\mathrm{R} \$ 4,00$. Pela sugestão dos especialistas, e considerando um prêmio mínimo de $50 \%$ pelo produto sem agrotóxico, utilizou-se como intervalo de preço sugerido o mínimo de $\mathrm{R} \$ 3,00$ e máximo de $\mathrm{R} \$ 7,00$ pela embalagem de produto sem agrotóxicos.

A escolha de um prêmio, isto é, o percentual pago a mais sobre o mesmo produto convencional, relativamente elevado para o produto diferenciado, comparando-se com a literatura, reside no fato de que nenhum dos referidos trabalhos analisou a DAP por um produto que é, naturalmente, consumido de forma esporádica. Acredita-se, dessa forma, que o efeito de uma elevação do preço de morangos tenha um impacto relativamente menor na renda do consumidor do que o de produtos de primeira necessidade.

Para determinar o tamanho amostral, foi utilizada a fórmula apresentada por Gil (1991), tendo como referência a população de Viçosa/MG, erro amostral de $5 \%$ e intervalo de confiança de $95 \%$, totalizando amostra de $n=270$. 
Foram aplicados dois questionários semiestruturados distintos, um contendo a afirmação "Estudos de laboratório mostram que pesticidas podem causar problemas de saúde, como defeitos ao nascer, câncer, dentre outros" e outro sem a informação, de forma a ser avaliado seu efeito sobre a predisposição a pagar. Cada tipo de questionário (com e sem informação) correspondeu a 50\% do total, tendo sido aplicado de forma aleatória, em locais de grande circulação de pessoas, como supermercados e feiras livres, nos períodos da manhã, tarde e noite, em junho de 2010.

Para estimar a DAP individual, utilizou-se a técnica de referendo simples, ou pergunta fechada. Na prática, essa técnica consiste em fazer uma única pergunta à qual o consumidor responderá sim ou não, caso concorde ou discorde do pagamento do valor proposto. Este tipo de pergunta é muito utilizado porque admite menor possibilidade para os entrevistados tentarem defender seus interesses ou beneficiar-se de alguma forma, assemelhando-se ao processo de escolha a que o consumidor está habituado (CUNHA, 2006).

De posse dos indicadores discretos da referida DAP, pode-se utilizar o método de Hanemann (1984) para obter a DAP verdadeira desejada. Como a resposta dada pelos indivíduos no questionário é uma variável discreta e dicotômica, Hanemann (1984) sugere a utilização do modelo Logit para estimar, primeiro, a probabilidade de um indivíduo se dispor a pagar e, em seguida, o preço em que a probabilidade de obtenção de uma resposta SIM seja de 50\%, considerando o valor máximo que um indivíduo estaria disposto a pagar. Esse modelo se baseia na função de probabilidade logística acumulada, sendo representado da seguinte forma:

$$
P_{j}=P\left(Y_{j}=1\right)=F\left(X_{j} \beta\right)=\frac{1}{1+\theta^{-\left(X_{j} \beta\right)}}
$$

em que $P_{j}$ representa a probabilidade de aceitação do pagamento proposto pelo morango orgânico e $\left(1-P_{j}\right)$ a probabilidade de não aceitação do 
valor proposto; $X_{j} \beta$ é um índice que representa as características do entrevistado; e " $e$ ", a base dos logaritmos naturais.

Uma vez que $P_{j}$ é não observável, estima-se (1) por máxima verossimilhança, sendo que $X_{j} \beta$ assume a seguinte forma:

$X_{j} \beta=\beta_{0}+\beta_{1} X_{1}+\beta_{2} X_{2}+\ldots+\beta_{9} X_{9}+\varepsilon$

em que $x_{1}$, valor de pagamento sugerido aos entrevistados (R\$); $x_{2}$, renda familiar mensal do consumidor (em salários mínimos); $x_{3}$, nível de escolaridade do consumidor (anos de estudo); $x_{4}$, idade do consumidor; $x_{5}$, gênero do entrevistado $(0=$ feminino $1=$ masculino $) ; x_{\epsilon}$, consumo de morango $(0=$ não consumo ou esporadicamente e $1=$ semanalmente ou mensalmente); $x_{7}$, presença ou não de pessoas idosas - maiores de 65 anos; $x_{\mathrm{g}}$, presença ou não de pessoas jovens menores de 18 anos; $x_{\mathrm{g}}$, responsabilidade pela certificação de que o morango foi produzido sem agrotóxicos; e $\epsilon$ representa o erro aleatório.

As expectativas a priori sobre os sinais dos coeficientes relacionados às variáveis explicativas são as seguintes: $\beta_{1}$ negativo indica que a probabilidade de o consumidor aceitar o preço proposto decresce com o aumento do valor sugerido; $\beta_{2}, \beta_{3}$ e $\beta_{8}$ positivos significam que a probabilidade de aceitar o preço proposto cresce com o aumento de tais variáveis, sendo as expectativas formadas com base na literatura.

No caso do coeficiente $\beta_{6}$, espera-se um sinal positivo, pois é razoável supor que os consumidores que tenham o hábito de consumir morango tenham uma maior DAP em relação a ele do que aqueles que consomem morango esporadicamente. Com relação aos coeficientes $\beta_{4}, \beta_{7}$ e $\beta_{9}$, eles podem ou não ser positivos, uma vez que não há consenso na literatura 
sobre o sinal e a influência dessas características na DAP por produtos diferenciados.

Para uma amostra qualquer, a abordagem de Hanemann (1984) fornece apenas uma estimativa para a DAP verdadeira dos indivíduos. Nesse sentido, conforme observado por Cirino e Lima (2008), não há como calcular o erro-padrão de tal medida. Ainda de acordo com esses autores, a determinação de um desvio-padrão para a DAP verdadeira é importante para a análise de sua precisão estatística, fornecendo, dessa forma, subsídios para uma avaliação mais criteriosa de tal medida por parte daqueles que a utilizarão.

Para superar essa limitação, utilizou-se o método Bootstrap para obter o erro-padrão para a DAP verdadeira estimada. Beluzzo Jr.(1995) salienta que, embora existam metodologias alternativas para tal fim, o Bootstrap apresenta resultados satisfatórios quando se utiliza a distribuição logística, como ocorre neste trabalho.

O método do Bootstrap utilizado neste trabalho segue Cirino e Lima (2008) e Silva e Lima (2004). O método parte da estimação do modelo (2), do qual se obtém um vetor inicial de resíduos estimados. Embaralhando esses últimos, gera-se uma nova variável dependente, sendo que a estimativa de $Y_{j}$ para a primeira observação não precisa ser somada, necessariamente, com o primeiro resíduo estimado.

Repetindo $T$ vezes o procedimento, tem-se um conjunto diferente de $T$ variáveis aleatórias distintas e, consequentemente, de $T$ vetores de parâmetros $\beta$. Esses parâmetros fornecerão $T$ valores da DAP verdadeira, que serão utilizados para formar uma distribuição probabilística dessa medida, cujo ponto médio e o desvio-padrão serão, respectivamente, a DAP verdadeira dos entrevistados e o seu desvio-padrão. Neste trabalho, foram feitas 10.000 interações para determinar a DAP verdadeira média e seu desvio-padrão. 
Para estimar a verdadeira DAP pelo morango sem agrotóxico, foram estimados três modelos, como o proposto na equação (2), diferenciandose quanto à amostra utilizada em cada um deles. $\mathrm{O}$ primeiro modelo contempla todos os entrevistados na pesquisa, tendo as variáveis DAP, renda e idade se mostrado significativas. O segundo modelo, denominado sem informação, compreende os questionários em que os entrevistados não receberam, a priori, as informações dos malefícios dos pesticidas. Nesse modelo, apenas a variável DAP foi significativa. Por fim, no modelo com informação, verificou-se a significância estatística das variáveis DAP e renda familiar, ambas em nível de 1\% de significância. Para aumentar a precisão da estimativa da verdadeira DAP pelo morango sem agrotóxico, optou-se por reestimar os modelos Logit somente com as variáveis significativas.

Para verificar a presença de multicolinearidade entre as variáveis do modelo reduzido, foram empregados os testes do fator de inflação da variância (FIV) para cada uma das amostras apresentadas na Tabela 4.

\section{Resultados}

Do total de entrevistados, $54,8 \%$ eram do sexo masculino, $21,7 \%$ tinham idade entre 18 e 24 anos, 74,0 entre 24 e 65 anos e 3,6\% tinham mais de 65 anos (Tabela 1). Ainda, 58,93\% se disseram diretamente responsáveis pelas decisões de compra de alimentos em seus lares. Desses, 44,2\% eram do sexo masculino, 64,6\% tinham idade entre 24 e 64 anos. No geral, $7,5 \%$ dos entrevistados tinham ensino fundamental completo, $15,7 \%$ dos entrevistados, ensino médio completo, e 13,2\%, nível superior completo. 
Tabela 1. Características dos entrevistados no município da Zona da Mata Mineira. 2010.

\begin{tabular}{ccccc}
\hline Variável & \multicolumn{4}{c}{ Proporção (\%) } \\
\hline Gênero do entrevistado & $\begin{array}{c}\text { Masculino } \\
54,84\end{array}$ & \multicolumn{3}{c}{ Feminino } \\
& & & 45,16 & \\
Idade do entrevistado (anos) & $\mathbf{1 8 - 2 4}$ & $\mathbf{2 4 - 6 5}$ & $\geq 65$ & \\
& 21,66 & 74,01 & 3,61 & \\
& & & & \\
Decisão da compra de alimentos & Entrevistado & Cônjuge & Outros & Ambos \\
& 58,93 & 15,36 & 18,21 & 7,5 \\
\hline
\end{tabular}

Fonte: resultado da pesquisa.

Considerando a totalidade dos entrevistados, $61,4 \%$ afirmaram consumir morango esporadicamente, enquanto apenas 3,6\% dos entrevistados consumiam morango semanalmente. Quando questionados sobre os fatores considerados muito importantes para o consumidor no momento da compra, 32,1\% dos entrevistados citaram "Preço baixo" e $48,1 \%$ apontaram "Proteção ao meio ambiente". Entretanto, os fatores com maior destaque foram "Saúde" (Segurança Alimentar) e "Qualidade", mencionados como muito importantes por 71,4\% e 81,1\% dos entrevistados, respectivamente. Esses resultados indicam que os consumidores dão um elevado grau de importância a outros aspectos dos produtos que não o preço.

A maioria dos entrevistados $(46,4 \%)$ afirmou confiar mais se a certificação referente à ausência de agrotóxicos no produto fosse de responsabilidade dos produtores, sendo que $36,4 \%$ confiariam mais se fosse responsabilidade do governo e 17,3\% atribuíram tal responsabilidade às certificadoras privadas.

Com relação à verdadeira DAP pelo morango sem agrotóxico, foram estimados três modelos (Tabela 2). 
Tabela 2. Resultados dos modelos Logit reduzidos para estimação da DAP real para o morango sem agrotóxico. 2010.

\begin{tabular}{cccc}
\hline Variável & $\begin{array}{c}\text { Total de } \\
\text { Entrevistados }\end{array}$ & $\begin{array}{c}\text { Sem } \\
\text { Informação }\end{array}$ & $\begin{array}{c}\text { Com } \\
\text { Informação }\end{array}$ \\
\hline Intercepto & $6,1021^{* * *}$ & $6,8881^{* * *}$ & $5,4899^{* * *}$ \\
DAP & $(0,9377)$ & $(1,5908)$ & $(1,4113)$ \\
Renda & $-0,8784^{* * *}$ & $-0,9949 * * *$ & $-0,7985^{* * *}$ \\
& $(0,1654)$ & $(0,2495)$ & $(0,2616)$ \\
Idade & $0,5431^{* * *}$ & 0,3497 & $0,7895 * * *$ \\
& $(0,1835)$ & $(0,2831)$ & $(0,2890)$ \\
Escolaridade & $-0,2442^{*}$ & $-0,0283$ & $-0,1776$ \\
& $(0,0126)$ & $(0,1943)$ & $(0,0217)$ \\
Teste LR & $-0,1141$ & $-0,0450$ & $-0,2047$ \\
Prob. $>$ LR & $(0,0720)$ & $(0,1041)$ & $(0,1343)$ \\
Pseudo $\mathrm{R}^{2}$ & 34,5060 & 19,1660 & 20,0780 \\
Prob. Média & $(0,0000)$ & $(0,0010)$ & $(0,0000)$ \\
Prev. Corretas & 0,1304 & & $87,75 \%$ \\
Obs. Com (Y=0) & $85,86 \%$ & $85,12 \%$ & $82,22 \%$ \\
Obs. Com (Y=1) & $79,78 \%$ & $79,58 \%$ & 23 \\
Obs. Total & 52 & 29 & 112 \\
\hline
\end{tabular}

Fonte: Resultados da pesquisa.

Nota: Entre parênteses está o desvio-padrão dos coeficientes. $* * *$ significativo a $1 \%, * *$ significativo a $5 \%, *$ significativo a $10 \%$. DAP = disposição a pagar

Sobre a qualidade estatística dos modelos reduzidos, observou-se que o teste da razão de verossimilhança - um teste de significância global do modelo que consiste em testar a hipótese nula de uma regressão restrita contra uma irrestrita - foi altamente significativo nos três modelos. Dessa forma, pode-se dizer que as regressões entre a probabilidade de um indivíduo responder positivamente à valoração do morango sem agrotóxico e às variáveis explicativas consideradas podem ser utilizadas para fazer inferências estatísticas.

Quanto ao poder de previsão, observou-se que os modelos apresentaram resultados satisfatórios e semelhantes, visto que 79,78\%, 79,58\% 
e $82,28 \%$ das previsões dos modelos, total de entrevistados, sem informação e com informação, respectivamente, estavam corretas. A probabilidade média de aceitação de um preço não-negativo relativamente à aquisição de morango sem agrotóxico foi estimada em 85,86\%, $85,12 \%$ e $87,75 \%$. Tal situação indica, a priori, uma tendência de que seja revelada uma preferência por parte dos entrevistados, no sentido de uma DAP verdadeira significativa, em relação a um desejo concreto de consumir morango sem agrotóxico. Os FIVs aproximam-se da unidade, indicando que nenhuma das variáveis apresenta multicolinearidade elevada (Tabela 3).

Tabela 3. Teste de Multicolinearidade entre as variáveis explicativas do modelo Logit reduzido para estimação da DAP real do morango sem agrotóxico. 2010.

\begin{tabular}{cccc}
\hline Variável & $\begin{array}{c}\text { FIV - Total de } \\
\text { Entrevistados }\end{array}$ & $\begin{array}{c}\text { FIV - Sem } \\
\text { Informação }\end{array}$ & $\begin{array}{c}\text { FIV - Com } \\
\text { Informação }\end{array}$ \\
\hline DAP $_{\mathrm{p}}$ & 1,02 & 1,01 & 1,05 \\
Renda & 1,41 & 1,65 & 1,24 \\
Idade & 1,36 & 1,45 & 1,30 \\
Escolaridade & 1,55 & 1,72 & 1,43 \\
\hline
\end{tabular}

Fonte: Resultados da pesquisa Nota: DAP = disposição a pagar; FIV = fator de inflação da variância

A Tabela 4 apresenta o valor dos efeitos marginais de cada uma das variáveis explicativas sobre a probabilidade de o entrevistado aceitar pagar mais pelo morango sem agrotóxico, para cada um dos três modelos. A análise do significado estatístico de cada uma das variáveis é feita com base nesses efeitos marginais, que foram calculados no ponto médio da amostra. 
Tabela 4. Efeito marginal do modelo Logit reduzido para estimação da DAP real para o morango sem agrotóxico. 2010.

\begin{tabular}{cccc}
\hline Variável & $\begin{array}{c}\text { Total de } \\
\text { Entrevistados }\end{array}$ & $\begin{array}{c}\text { Sem } \\
\text { Informação }\end{array}$ & $\begin{array}{c}\text { Com } \\
\text { Informação }\end{array}$ \\
\hline DAP $_{\mathrm{p}}$ & $-0,1066^{* * *}$ & $-0,1261^{* * *}$ & $-0,0858^{* * *}$ \\
& $(0,0206)$ & $(0,0302)$ & $(0,0320)$ \\
Renda & $0,0659^{* * *}$ & 0,0443 & $0,0848^{* * *}$ \\
& $(0,0211)$ & $(0,0343)$ & $(0,0294)$ \\
Idade & $-0,0029^{*}$ & $-0,0036$ & $-0,0019$ \\
& $(0,0015)$ & $(0,0246)$ & $(0,0024)$ \\
Escolaridade & $-0,1386$ & $-0,0057$ & $-0,0220$ \\
& $(0,0090)$ & $(0,0132)$ & $(0,0143)$ \\
\hline
\end{tabular}

Fonte: Resultados da pesquisa.

Nota: Entre parênteses está o desvio-padrão dos coeficientes. *** significativo a $1 \%, * *$ significativo a $5 \%, *$ significativo a $10 \%$. DAP $=$ disposição a pagar

O efeito marginal da variável DAP proposta sobre a probabilidade de o entrevistado aceitar pagar pelo morango sem agrotóxico foi negativo nos três modelos, coerente com uma relação de demanda, como a que se busca neste trabalho. Tal efeito indica que um aumento de uma unidade na DAP proposta, mantida as demais variáveis constantes, diminui a probabilidade de pagamento pelo morango sem agrotóxico em 0,1066 pontos percentuais no modelo que contém a totalidade dos entrevistados, em 0,1261 pontos percentuais no modelo sem informação e em 0,0858 pontos no modelo com informação.

Esses resultados mostram a importância da informação a respeito dos malefícios dos agrotóxicos na DAP pelo produto. Nos questionários que apresentavam a afirmação de que "Estudos de laboratório mostram que pesticidas podem causar problemas de saúde, como defeitos ao nascer, câncer, dentre outros", o efeito marginal negativo da variável DAP pelo produto sem agrotóxico sobre a probabilidade de o entrevistado aceitar pagar mais pelo produto foi menor.

O efeito marginal da variável renda familiar também se mostrou de acordo com o que sugere a teoria, uma vez que um aumento de 
uma unidade monetária na referida variável eleva em 0,0659 e em 0,0848 pontos percentuais a probabilidade de uma resposta positiva à aquisição do produto em questão, respectivamente, nos modelos total de entrevistados e com informação, os quais apresentaram esta variável significativa estatisticamente. Considerando um aumento de $\mathrm{R} \$ 1.000,00$ na renda familiar, a elevação sobre a probabilidade considerada seria de 65,90 e de 84,80 pontos percentuais em cada um dos modelos citados, respectivamente, indicando que a referida variável tem influência importante sobre o fenômeno em estudo.

Em relação à idade, houve um efeito marginal negativo, embora pequeno, e significativo apenas para o modelo com o total de entrevistados, indicando que uma elevação em um ano de vida reduz a probabilidade de pagamento pelo morango sem agrotóxico em 0,0029 pontos percentuais. Já a variável escolaridade foi negativa e não se mostrou significativa em nenhum dos três modelos.

Para obter a verdadeira DAP pelo morango sem agrotóxico com maior precisão, utilizou-se o método Bootstrap, cujos resultados são apresentados na Tabela 5 .

Tabela 5. Estimação da Disposição a pagar pelo morango sem agrotóxico, método Bootstrap, em R $\$$.

\begin{tabular}{cccc}
\hline DAP $_{\mathbf{r}}$ & $\begin{array}{c}\text { Total de } \\
\text { Entrevistados }\end{array}$ & $\begin{array}{c}\text { Sem } \\
\text { Informação }\end{array}$ & $\begin{array}{c}\text { Com } \\
\text { Informação }\end{array}$ \\
\hline Média & 7,0544 & 6,6885 & 7,7665 \\
Desvio-padrão & 0,3932 & 0,4132 & 0,8691 \\
\hline
\end{tabular}

Fonte: Resultados da pesquisa.

Nota: DAP = disposição a pagar

Utilizando-se as estimativas dos modelos reduzidos e a abordagem de Hanemann (1984), foram obtidas as seguintes DAPs médias verdadeiras: $\mathrm{R} \$ 7,05$ para o modelo contendo todas as entrevistas, $\mathrm{R} \$ 6,68$ para o 
modelo sem informação e R\$ 7,76 para o modelo com informação. O valor do desvio-padrão de cada uma das DAPs indica que o valor médio dessa medida é uma boa representação da DAP verdadeira, uma vez esses valores são pequenos, significando que as distribuições das DAPs estão concentradas em torno de suas respectivas médias.

\section{Discussão}

A grande participação dos homens na decisão de compra de alimentos chama atenção, pois, como observado por Stager e Witte (1989), citados por Mendes (2002), a literatura sugere que as mulheres são mais preocupadas com a saúde do que os homens e são socializadas para os afazeres do lar. Este resultado pode estar relacionado a mudanças socioeconômicas da população brasileira, em que a grande inserção das mulheres no mercado de trabalho tem exigido maior participação dos homens em tarefas antes rotuladas como exclusivamente femininas.

Considerando o valor médio $(\mathrm{R} \$ 7,05)$ e o preço médio de mercado para um pacote de morango de $300 \mathrm{~g}$ igual a $\mathrm{R} \$ 4,00$, o prêmio pago pelo produto sem agrotóxicos foi de $76,2 \%$. Os valores da DAP encontrados são significativamente maiores do que os descritos em trabalhos anteriores, como os de Misra, Huang e Ott (1991), Boccaletti e Nardella (2000) e Magnusson (2002), em que o prêmio pago pelos produtos sem agrotóxicos foi em média de $10 \%$ do convencional.

A diferença entre a DAP estimada com base nos coeficientes do modelo contendo todas as entrevistas e a DAP estimada como base nos coeficientes do modelo contendo apenas as entrevistas com a referida informação foi de aproximadamente $\mathrm{R} \$ 0,72$, não sendo uma diferença muito significativa. No entanto, quando se compara a DAP estimada com base nos coeficientes do modelo sem informação com a DAP estimada como base nos coeficientes do modelo com informação, a diferença entre elas é de, aproximadamente, $\mathrm{R} \$ 1,10$. 
Esses resultados reforçam a importância da informação a respeito dos malefícios dos agrotóxicos sobre a saúde humana sobre a DAP dos consumidores por produtos sem essas substâncias. Também ficou evidente que, apesar de toda informação disponível na mídia sobre o efeito dos agrotóxicos sobre a saúde, a maioria dos consumidores entrevistados ainda desconhece as implicações da ingestão destas substâncias para a saúde.

Os valores das DAP são coerentes com o intervalo de valores sugeridos pela pesquisa de mercado e pelas entrevistas com especialistas na produção e comercialização de morangos orgânicos, sendo definido entre $\mathrm{R} \$ 3,00$ a $\mathrm{R} \$ 7,00$. Entretanto, os valores estimados foram bem mais elevados do que o valor médio de $\mathrm{R} \$ 4,97$, obtido pela aplicação dos questionários.

Os resultados encontrados em estudo realizado por Misra, Huang e Ott (1991), que objetivavam estimar a DAP de consumidores por produtos frescos e sem agrotóxicos, em uma amostra de 379 pessoas na cidade de Geórgia (EUA), mostraram que 46\% dos entrevistados estavam dispostos a pagar mais por aquele tipo de produto, e que a maioria (87\%) não estaria disposta a pagar mais de $10 \%$ sobre o preço normal do produto. Por sua vez, Boccaletti e Nardella (2000) encontraram que a DAP por frutas e hortaliças frescas produzidas sem agrotóxicos se mostrou positivamente relacionada com a renda e com o nível de educação. Neste trabalho, foram entrevistados 336 consumidores, em três grandes supermercados do nordeste da Itália com, aproximadamente, 34\% dos entrevistados dispostos a pagar entre 6 e $10 \%$ a mais pelos produtos diferenciados e $11 \%$ dos entrevistados, mais de $20 \%$.

Em pesquisa feita por Magnusson (2002), 320 consumidores canadenses responderam questionário enviado pelo correio referente à DAP por diversos produtos processados e frescos. O nível de escolaridade mostrouse positivamente relacionado para a maioria dos produtos, enquanto não se pôde atestar com precisão a relação com a renda devido às abstenções sobre a questão. Os resultados mostraram que uma DAP de 5 a 20\% teve um efeito marginal positivo e significativo. 
Mendes (2002), por sua vez, avaliou a DAP e seus condicionantes para produtos hortifrutícolas no município de Piracicaba-SP, utilizando uma amostra de 316 consumidores. Os resultados mostraram correlação estatisticamente significativa e positiva entre variáveis de grupo de risco e atitude do consumidor em relação ao consumo de produtos livres de agrotóxicos. Entretanto, diferentemente dos resultados encontrados em outros trabalhos similares, fatores como renda, idade e escolaridade não foram significativos para explicar a DAP.

\section{Conclusões}

A preocupação com a alimentação saudável é uma das tendências comportamentais que surgem à medida que o nível educacional e de renda da população se elevam. Após a satisfação das necessidades básicas de alimentação, o próximo passo é a exigência pela oferta de alimentos nutritivos, seguros e sem agrotóxicos.

De acordo com os resultados, os consumidores participantes deste estudo estão dispostos a pagar quase o dobro por morangos produzidos sem agrotóxicos, no equivalente a $\mathrm{R} \$ 6,68$. No entanto, quando informados acerca dos malefícios do consumo dessas substâncias à saúde, a DAP aumentou aproximadamente $16 \%$, chegando a RS 7,76.

A diferença da DAP entre os consumidores que possuíam ou não informação sobre os malefícios dos agrotóxicos, por um lado, mostra a necessidade de os consumidores serem esclarecidos sobre os efeitos adversos dessas substâncias, por outro lado, mostra a importância dessa informação na decisão de compra do consumidor.

Portanto, pode-se observar que a preferência por morangos sem agrotóxicos bem como a preocupação com a saúde e a qualidade do alimento são fatores que influenciam a DAP pelos consumidores. 


\section{Referências}

AGÊNCIA NACIONAL DE VIGILÂNCIA SANITÁRIA (ANVISA). MINISTÉRIO DA SAÚDE. Programa de Análise de Resíduos de Agrotóxicos em Alimentos (PARA) relatório de atividades de 2010. Brasília-DF: Ministério da Saúde. 2011. 26p.

BELLUZZO JR W. Valoração de bens públicos: o método de valoração contingente. 1995. 115f. Dissertação (Mestrado em Economia). Universidade de São Paulo; São Paulo; 2006.

BOCCALETTI S; NARDELLA M. Consumer willingness to pay for pesticide-free fresh fruit and vegetables in Italy. International Food and Agribusiness Management Review v.3; n.3; p.297-310; 2000.

CARSON RT; FLORES NE; MEADE NF. Contigent valuation: controversies and evidence. Environmental and Resource Economics, v.19; p.173-210; 2001.

CIRINO JF; LIMA JE. Valoração contingente da Área de Proteção Ambiental (APA) de São José - MG: um estudo de caso. Rev. Econ. Sociol. Rural, v.46; n.3; p.647-72; 2008.

CUNHA CF. Disposição a pagar pelo café orgânico: um estudo no município de São Paulo. 2006. 166p. Tese (Doutorado em Economia Aplicada). Universidade de São Paulo - Escola Superior de Agricultura "Luiz de Queiroz"; São Paulo. 2006.

FUNDAÇÃO JOÃO PINHEIRO (FJP). Renda per capita dos municípios de Minas Gerais. Disponível em: <http://www.fjp.gov. br/index.php/servicos/82-servicos-cepp/1423-tabelas-sintese-de-rpcmunicipios-2010xls $>$. Acesso em 11 de dez. de 2012.

GIL AC. Téenicas de pesquisa em economia. São Paulo. Ed. Atlas. 1991. 195p. 
HANEMANN WM. Welfare Evaluations in Contingent Valuation Experiments with Discrete Responses. Am. J. Agri. Econ. v.66; n.3; p.332-41; 1984.

HENSON S; CASWELL J. Food safety regulation: an overview of contemporary issues. Food Policy, v.24; p.589-603; 1999.

MADAIL JCM; ANTUNES LE; BELARMINO LC et al. Avaliação econômica dos sistemas de produção de morango: convencional, integrado e orgânico. Pelotas: Embrapa. Comunicado técnico 181. 2007. Disponível em: <http:/www.agencia.cnptia.embrapa.br/Repositorio/ avaliacao_economica_000giwzwdzv02wx5ok05vadr129jqgbk.pdf $>$. Acesso em 10 de janeiro de 2013.

MAGNUSSON JE. Consumer interest in and willingness-to-pay for pesticide free production food products: a probit analysis. Canada: University of Manitoba, 2002. 356p.

MENDES CM. Disposição de pagar por alimentos seguros: o caso dos hortifrutícolas sem resíduos de agrotóxicos. 2002. 71f. Tese (Doutorado em Economia Aplicada). Universidade de São Paulo - Escola Superior de Agricultura “Luiz de Queiroz”; São Paulo. 2002.

MISRA SK; HUANG CL; OTT SL. Consumer willingness to pay for pesticide-free fresh produce. Western Journal of Agricultural Economics v.16; n.2; p.218-27; 1991.

MITCHELL RC; CARSON RT. Using surveys to value public goods: the contingent valuation method. Washington, D.C.: Resources for the Future; Distributed worldwide by the Johns Hopkins University Press, 1989. 463p.

PRESCOTT J; YOUNG A. Does information about MSG (monosodium glutamate) content influence consumer ratings of soups with and without added MSG? Appetite, v.39, p.25-33; 2002. 
ROZANA; STANGER A; WILLINGER M. Willingness-to-pay for food safety: an experimental investigation of quality certification on bidding behaviour. European Review of Agricultural Economics, v.31; n.4; p.409-25; 2004.

SEGERSON K. Mandatory versus voluntary approaches to food safety. Agribusiness, v.15; n.1; p.53-70; 1999.

SILVA RG; LIMA JE. Valoração contingente do parque "Chico Mendes": uma aplicação probabilística do método Referendum com bidding games. Rev. Econ. Sociol. Rural [online]; v.42; n.4; p.685-708; 2004 